\title{
La rencontre analytique dans les dispositifs à mediations thérapeutiques, aux limites de I'analyse
}

Anne Brun*1

Les médiations thérapeutiques en pratiques institutionnelles relèvent de la psychothérapie psychanalytique quand elles sont fondées sur la prise en compte du transfert et de l'associativité psychique, au fondement de la méthode analytique. A l'appui de la clinique de la psychose infantile, cet article interrogera les modalités particulières des processus de symbolisation qui s'y déploient, à partir notamment de la prise en compte de l'associativité sensorimotrice.

Mots clés: Associativité sensori-motrice, métapsychologie des médiations thérapeutiques, sensori-motricité, symbolisation primaire

${ }^{* 1}$ Université Lumière (Lyon, France). 


\section{ARTIGO}

Les pratiques des médiations thérapeutiques, comme la terre, la peinture, la musique, sont anciennes dans l'histoire du soin, notamment dans la psychothérapie psychanalytique des enfants et des psychoses, mais il semble en revanche novateur de tenter de les modéliser dans une théorisation d'ensemble référée à la théorie psychanalytique (Brun, 2009). Le premier constat est que la théorie comme la pratique de ces médiations a été traditionnellement négligée par les psychanalystes: les analystes contemporains ont en effet fort peu conceptualisé directement les dispositifs à médiation, ils évoquent la plupart du temps des cadres de psychothérapies classiques, fondés sur la parole, et il faut donc transposer, réinventer leur théorisation pour dégager la spécificité des processus en jeu dans les médiations. Les dispositifs cliniques classiques ou standard présentent en effet des limites quand il s'agit de travailler par exemple avec l'autisme, la psychose, la criminalité ou l'anti-socialité grave. Les dispositifs thérapeutiques à médiation concernent particulièrement les formes de pathologies du narcissisme et de l'identité, définies comme «situations limites de la subjectivité» ou «cliniques de l'extrême». ${ }^{1}$ À partir de ce premier constat, il s'agit donc de proposer une théorie générale des dispositifs à médiation, de modéliser ces dispositifs de soin susceptibles de traiter la destructivité majeure de patients en souffrance avec la symbolisation. ${ }^{2}$

Un second constat est que les psychanalystes s'aventurent très peu sur ce terrain, et, dans le cadre de la psychothérapie institutionnelle, la plupart des groupes à médiation sont animés par des infirmiers, des éducateurs, des intervenants parfois dénommés art thérapeutes, encore trop peu par des psychologues référés à

${ }^{1}$ Le terme «Cliniques de l'extrême», forgé à partir du terme de «situations extrêmes» avancé par Bettelheim à propos de son expérience des camps de concentration, a été proposé et exploré par R. Roussillon: il désigne les pathologies narcissiques identitaires de sujets en difficulté majeure pour accéder aux processus de symbolisation (Roussillon, 1991, 2005, 2007).

${ }^{2}$ Telle a été notre perspective dans deux ouvrages, Mediaciones terapeuticas y psicosis infantil, Barcelone, éd. Herder, 413 p, 2007, trad. 2009, et un ouvrage très récent, Manuel des médiations thérapeutiques, Paris, Dunod, 2013, coécrit avec B. Chouvier et R. Roussillon. 
l'épistémologie psychanalytique, même si cela évolue actuellement. Enfin, troisième constat, même si l'on assiste actuellement un essor considérable de ces dispositifs à médiation, réputés efficaces, il s'agit davantage de transmettre des pratiques plutôt que d'élaborer une modélisation psychanalytique, susceptible de définir la nature des processus de transformation mis en jeu dans la dynamique thérapeutique: en revanche, on dispose de théorisations pour la pratique psychanalytique des groupes, tant pour les adultes que pour les enfants.

Cette réflexion vise notamment à dégager les facteurs de symbolisation dans les dispositifs de soin à médiation pour la psychopathologie lourde, mais, dans le cadre restreint de cet article, je limiterai mes exemples au champ de la psychose infantile et de l'autisme, où les médiations thérapeutiques constituent un cadre de soin privilégié. La question sera de savoir s'il est possible de proposer une métapsychologie de la médiation dans la psychose infantile, fondée certes sur la métapsychologie freudienne mais aussi sur d'autres types de théorisation dans l'histoire de la psychanalyse.

\section{Médiations thérapeutiques et associativité sensorimotrice}

Pour pouvoir inscrire les médiations dans le champ de la psychothérapie psychanalytique, et non pas sous la bannière de ce que l'on désigne communément par art thérapie, il s'impose d'abord de prendre en compte l'associativité, au fondement de la méthode psychanalytique. Dans le cadre des médiations thérapeutiques, le repérage d'un processus associatif concerne classiquement les associations du patient en lien avec sa production, ainsi que les chaînes associatives groupales, dans le cadre d'un groupe. Mais, pour des pathologies en difficulté majeure avec le langage verbal, il ne faut pas oublier d'autres types de langage, comme le langage $\mathrm{du}$ corps et le langage de l'acte, présents dans l'oeuvre freudienne, comme l'a montré notamment Roussillon (2008). C'est en effet une forme «d'associativité» non verbale qui se déploie avec le médium, une associativité liée au geste, à la succession des séquences d'utilisation, à l'enchaînement des formes et aux déformations que le sujet fait subir au medium. Le clinicien se centre souvent sur la production finale, sur la forme produite par le sujet avec le médium, alors qu'on a tout intérêt à suivre «pas à pas» tout le processus de production lui-même, l'intégralité du jeu avec les diverses propriétés sensorielles du médium. Les thérapeutes, psychologues cliniciens et/ou psychanalystes, doivent en quelque sorte réorienter leur capacité d'écoute du côté de la prise en compte du langage sensorimoteur.

De quoi se compose cette associativité non verbale? Le clinicien sera attentif à la gestualité des patients, à leurs mimiques, à leurs postures, à toute la dynamique 


\section{ARTIGO}

mimogestuoposturale, mais aussi à leurs choix de tel ou tel instrument pour travailler le medium, de tel ou tel matériau ou support, de telle ou telle technique et la façon dont s'enchaîne au fil des ateliers thérapeutiques toute cette dynamique sensorimotrice pour chaque patient et pour le groupe: c'est essentiel car l'écoute de l'associativité psychique est au fondement de la méthode analytique. De façon générale, l'intérêt de ces thérapies à médiation consiste en effet à considérer la sensori-motricité comme un vecteur de symbolisation.

On évoque par exemple souvent l'extrême difficulté des enfants psychotiques à entrer dans le registre symbolique et à pouvoir accéder aux processus de représentation, du fait de la prédominance du registre sensorimoteur qui obèrerait les processus de symbolisation. On risque alors de s'engager dans une perspective déficitaire peu mobilisatrice sur le plan thérapeutique: cet article développera au contraire l'idée que c'est justement à partir de la sensori-motricité qu'une dynamique de symbolisation pourra s'enclencher, du fait de la rencontre de l'enfant avec la matérialité du medium malléable, qui sera inscrite dans le champ transférentiel. Les dispositifs de médiations thérapeutiques permettent en effet aux enfants psychotiques et autistes d'engager des modalités spécifiques de symbolisation, qui ne passeront pas d'abord par le langage verbal, avec lequel ces enfants sont souvent en difficulté, mais qui prendront en compte d'autres formes de langage, comme le langage de la motricité. Le travail thérapeutique s'effectuera donc à partir de la sensori-motricité de l'enfant, mais aussi des qualités sensorielles de l'objet médiateur et de la mise en jeu par les thérapeutes d'un langage sensorimoteur.

L'associativité ne peut être prise en compte que dans un cadre-dispositif qui relève de la psychothérapie psychanalytique, donc fondé sur la dynamique transféro-contre-transférentielle: dans cette perspective, comment spécifier le transfert dans ces dispositifs à médiation référés à la psychothérapie psychanalytique? D'abord par le fait que le transfert s'effectue sur le medium malléable, tel que Marion Milner (1955/1979) l'a défini, qui désigne à la fois le matériau, la matière, à la fois le thérapeute. Le thérapeute est le représentant du medium, comme le medium est le représentant du thérapeute. L'objet médiateur n'est donc pas thérapeutique en soi, cela dépend du cadre et du dispositif.

Pour que l'associativité sensorimotrice liée à l'utilisation du medium soit optimale, il faut laisser la plus grande liberté possible dans l'utilisation du medium.

\section{La règle fondamentale}

Les cliniciens énoncent la règle fondamentale, autre élément essentiel dans la méthode psychanalytique, sur le modèle de la règle fondamentale formulée à l'orée 
d'une cure psychanalytique. Cette règle fondamentale, organisatrice du cadre thérapeutique à médiation, comportera de façon centrale la référence à l'objet médiateur, ainsi qu'à l'association libre. On pourra dire, par exemple dans le cadre de la médiation picturale:

Vous pouvez dire ou faire avec les matériaux à votre disposition ici tout ce qui vous vient à l'esprit, comme cela se présente à vous, sans critique ni restriction, même si cela vous paraît futile, absurde ou déplaisant. Vous pouvez utiliser tout ce qui est là pour faire de la peinture (ou autre), comme vous voulez. Soyez aussi spontané que possible.

La liberté est paradoxalement prescrite mais elle est aussi une sorte d'objectif à atteindre, comme dans la règle classique, la possibilité de se laisse aller à l'association libre avec le medium. Une différence essentielle avec la formulation classique de cette règle est évidemment la référence au faire avec le medium, ouvrant le champ à l'associativité sensorimotrice.

Avec les enfants, la formulation de la règle fondamentale s'impose aussi, en adaptant l'énoncé selon les enfants concernés:

On est là pour dire avec la peinture (ou la terre, la musique, les marionnettes etc.) et avec les mots ce qui se passe dans notre tête, ce qui est difficile, ce qui fait mal, ce qui fait peur, et aussi ce qui fait plaisir. On peut raconter avec la peinture (ou autre) et on peut parler. Vous (ou tu) pouvez utiliser tout ce qui est là pour faire de la peinture (ou autre), comme vous voulez.

Dans la formulation de cette règle, inspirée de Haag, l'idée de la liberté dans l'utilisation du médium apparaît fondamentale: dans ce contexte, il s'agit principalement de laisser les enfants utiliser à leur gré l'ensemble du matériel mis à leur disposition. Ceux-ci choisissent leur façon de peindre, leur matériel et leurs techniques, qui peuvent éventuellement leur être présentées mais l'expérience montre qu'un enfant ne se saisit jamais d'une technique, si elle ne lui permet pas de travailler un aspect de sa problématique. Les thérapeutes se laissent utiliser par les enfants, sans leur demander de représentation et sans peindre avec eux, sauf occasionnellement, quand cela présente un intérêt thérapeutique.

Dans ce contexte, il s'agit principalement de laisser les patients, adultes ou enfants, utiliser à leur gré l'ensemble du matériel mis à leur disposition. Ceux-ci choisissent leur façon de peindre, de modeler, d'utiliser le medium, leur matériel et leurs techniques, qui peuvent éventuellement leur être présentées mais l'expérience montre qu'un patient ne se saisit jamais d'une technique, si elle ne lui permet pas de travailler un aspect de sa problématique. Les thérapeutes se laissent utiliser par les patients, sans leur demander de représentation et sans travailler le medium matérialisé avec eux, sauf occasionnellement, quand cela présente un intérêt thérapeutique. Dans certains cas, comme dans les groupes thérapeutiques d'écriture, 


\section{ARTIGO}

un des cliniciens peut participer au travail du medium, écrire ou modeler etc., à condition qu'il garde présent à l'esprit que sa production est une interprétation concrète, une figuration destinée au groupe, un reflet de la fantasmatique groupale inconsciente.

\section{Transfert sur le medium malléable}

C'est la prise en compte par les thérapeutes de ce libre jeu associatif qui va permettre le déploiement du champ transférentiel, le transfert étant le second fondement de la méthode analytique. Quelle sera la spécificité du transfert dans ces dispositifs à médiation référés à la psychothérapie psychanalytique? D'abord le fait que le transfert s'effectue sur le medium malléable, tel que Marion Milner l'a défini, qui désigne à la fois le matériau, la matière, à la fois le thérapeute. Le thérapeute est le représentant du medium, comme le medium est le représentant du thérapeute. L'objet médiateur n'est donc pas thérapeutique en soi, cela dépend du cadre et du dispositif. Il s'agit donc d'un transfert sur la matérialité du medium qui va devenir du coup une «matière à symbolisation» (Chouvier et coll., 2002).

Il s'impose d'abord de différencier deux types principaux de dispositifs, aux enjeux fondamentalement différents, bien que le travail à partir d'un medium malléable soit leur principe commun: il s'agit des dispositifs de médiation à création et les dispositifs thérapeutiques à médiation. Les ateliers à création se présentent souvent comme «ouverts», et certains donnent lieu à des expositions de productions: ils peuvent être animés par des infirmiers, des soignants non psychologues ou psychologues, éventuellement par des artistes, plasticiens ou musiciens, sans formation à la psychologie ni à la psychanalyse.

Ces cadres-dispositifs de médiations à création ne sont en effet ni fondés sur l'exploitation du transfert ni sur une interprétation des processus à l'œuvre, mais leurs enjeux concernent un accompagnement du travail des productions, ainsi qu'une centration sur la capacité de créer et de transformer des formes. Dans un tel cadre, l'accent mis sur la mise en mots s'avère variable, l'attention portée à la dynamique de l'association libre et aux chaînes associatives groupales ne se présentant pas comme une précondition de ce type de dispositif. Ces ateliers à création ne relèvent donc pas d'une pratique directement référée à la psychothérapie psychanalytique, mais ils peuvent enclencher une dynamique de symbolisation, qui a une portée thérapeutique certaine.

Quant aux dispositifs thérapeutiques à médiation, ils relèvent au contraire du champ de la psychothérapie psychanalytique, par la prise en compte du transfert, focalisé par l'objet médiateur, et ils permettent d'interroger les enjeux 
transférentiels, comme la dynamique psychique sous jacente. Ces dispositifs sont centrés, comme les dispositifs artistiques à création, sur la production de figurations, de représentations, de symboles, mais cette production est cette fois subordonnée à l'objectif de dégager les enjeux conscients et inconscients des figurations et représentations produites. Ils sont donc fondés sur la prise en compte de la dynamique transférentielle, focalisée par l'objet médiateur, ainsi que sur l'analyse des processus à l'œuvre dans le travail du médium malléable avec un décryptage des significations latentes; les groupes thérapeutiques d'expression sont fermés ou semi-ouverts, pour que le travail sur la fantasmatique groupale inconsciente et la réalité psychique de groupe soit possible, les productions ne sont pas exposées et restent dans l'intimité du groupe ou de la relation individuelle avec un ou deux thérapeutes. Ces dispositifs sont nécessairement animés ou supervisés par des professionnels de la psychologie.

Le transfert sur le médium malléable apparaît ainsi comme une modalité du transfert sur le cadre: ainsi, quand Marion Milner (1955/1979) définit le médium malléable, elle indique que l'enfant est capable de l'utiliser et d'utiliser la salle de jeu comme "cette substance malléable d'interposition". L'expérience montre que le transfert sur le medium, au double sens du matériau et du thérapeute, s'avère le transfert de la relation première de l'enfant à l'objet. L'enfant raconte par sa gestualité et son travail du medium son histoire, sa vie psychique, ses terreurs primitives, avant le langage verbal. C'est la réalité du lien précoce à l'objet qui va se transférer, se figurer et s'élaborer dans le cadre d'ateliers thérapeutiques individuels ou groupaux. Dans le cadre spécifique d'un groupe à médiation pour psychotiques et autistes, les enfants vont donc projeter les éléments morcelés de leur monde interne non seulement sur les thérapeutes et sur les autres sujets du groupe, selon le processus de diffraction du transfert dans un dispositif groupal conceptualisé par Kaës (1976/2000), mais aussi sur les éléments sensoriels du cadre thérapeutique. J'ai donc proposé de nommer ce processus «diffraction sensorielle» (Brun, 2007, pp. 63-66), puisque les fragments projetés sont diffractés en partie sur les éléments du cadre et notamment sur le medium malléable. Dans un groupe d'enfants psychotiques, cette diffraction du transfert n'opère donc pas seulement sur les membres du groupe et sur les thérapeutes, mais aussi sur les éléments du cadre matériel et sur l'objet médiateur.

Une partie du travail thérapeutique va s'effectuer à l'appui de l'impact des stimulations sensorielles provenant du matériel à disposition. La rencontre avec le medium malléable va donc activer chez l'enfant psychotique des sensations hallucinées, c'est-à-dire des vécus d'ordre psychocorporels, qui vont s'actualiser à partir des sensations procurées par la matérialité du medium. Ces sensations hallucinées vont pouvoir être mises en forme dans la matérialité du médiateur, en lien avec la dynamique transférentielle. 


\section{ARTIGO}

Comment définir ces sensations hallucinées? Elles renvoient souvent dans la psychose à des vécus originaires impensables, souvent des expériences d'agonie primitive décrites par Winnicott (1974), angoisses inimaginables du bébé, terreurs extrêmes, sans fin et sans limites, telles que le sujet se retire de cette expérience de mort psychique, pour pouvoir survivre. Du coup, ces expériences primitives catastrophiques n'ont jamais pu être représentées, car elles n'ont en quelque sorte pas été éprouvées par le sujet. Ces expériences non encore advenues peuvent justement se réactualiser et se figurer au gré de la rencontre avec tel ou tel matériau, telle ou telle technique, et il est impossible d'anticiper ce qui, dans la médiation picturale, va mobiliser chaque enfant de façon singulière et différente.

Par exemple, avec des enfants psychotiques, on constate souvent au début d'un groupe thérapeutique en peinture un acharnement de certains enfants à effacer toute trace sur leur feuille, à faire le vide, à diluer toutes les taches de peinture dans l'eau, jusqu'à l'effacement complet. Ce que l'enfant fait subir à la feuille indique ses angoisses, ici une mise en figure d'une terreur d'effacement, de disparition ou de dissolution. Celui-là exprime ainsi une terreur de néantisation, un vécu de mort psychique, contre lequel il va lutter, en infligeant à la feuille le vide, dans un processus de retournement passif actif. Le retournement passif/actif constitue souvent la première forme de symbolisation pour un enfant psychotique.

Cela peut être le trouage de la feuille, souvent en lien avec une angoisse d'effondrement, le lacérage, le déchiquetage de la feuille pour les enfants symbiotiques, en lien avec une terreur «d'être violemment pénétré dedans», qu'il retourne sur la feuille. Il arrive aussi que toute rencontre entre instrument, peinture et feuille ne puisse qu'aboutir à une destruction, par exemple le Je se présente comme «Moi/ feuille peau trouée ou percée ou arrachée». Il peut s'agir aussi d'une sensation d'arrachement d'une peau commune, en lien avec le décollage d'une peinture plastifiée, ou encore d'un vécu de glissade sans fin et de chute sur la feuille etc. «Moi/pinceau englouti dans la peinture». Ces sensations hallucinées proviennent d'une sorte d'effet miroir entre l'enfant et le medium malléable. L'enfant devient pour ainsi dire un Je/peinture qui se liquéfie, qui disparaît ou qui est aspiré par un trou, ou encore, en miroir avec la feuille de peinture, «un appui qui s'effondre» On constate une indifférenciation entre le corps de l'enfant, la matière picturale, les différents instruments. Dans la psychose, il s'agit d'une relation spéculaire entre l'enfant et le medium.

Ces dispositifs permettent de réactualiser des expériences primitives, d'ordre sensoriel, affectif et moteur, expériences non symbolisées, non inscrites dans l'appareil de langage. Comme ces dernières sont expérimentées avant l'apparition du langage verbal, elles se sont justement inscrites selon des modalités autres que langagières, tels que le langage du corps, le langage de l'affect, la mise en jeu de la sensori-motricité, particulièrement sollicités dans le cadre des médiations thérapeutiques. 
C'est la perception dans la réalité des sensations procurées par la matérialité du médiateur, la liquidité, le trouage ou l'arrachement de la feuille, qui activent le processus hallucinatoire chez l'enfant, et, réciproquement, l'enfant met en forme dans le matériau ses propres sensations hallucinées, liées à des expériences antérieures, qu'il associera aux sensations données par le médiateur. La médiation picturale permet de transformer la sensation hallucinée en une forme perceptive, la sensation hallucinée va prendre forme dans l'objet médiateur et devenir ainsi figurable et transformable : telle est la symbolisation primaire, conceptualisée par R. Roussillon (2001), comme le passage du perceptif à la représentation chose sensorielle.

Plus précisément le travail thérapeutique s'effectuera non seulement à partir de la matérialité du médiateur mais aussi à partir de la dynamique transférentielle. Le travail de mise en forme du medium va en effet susciter des messages corporels, visuels, kinesthésiques, mimo-gestuo-posturaux, qui vont pouvoir prendre sens dans les interrelations au sein de l'atelier, avec les thérapeutes mais aussi avec le groupe d'enfants. Le travail thérapeutique au sein de cadres-dispositifs à médiation amorcera ainsi un accès à la figurabilité par un processus de passage du registre sensori-moteur au figurable. Un des enjeux principaux des médiations thérapeutiques dans les cliniques de l'extrême consiste à pouvoir ainsi faire advenir à la figuration des expériences primitives non symbolisées, d'ordre sensori-affectivo-moteur.

\section{Un exemple clinique: la médiation picturale}

Dans la psychose infantile, le travail thérapeutique à partir de la médiation picturale s'articule autour de la constitution d'un fond pour la représentation. La peinture des enfants psychotiques se compose peu en effet de formes reconnaissables et identifiables, elle renvoie peu à des formes représentatives imagées, avec un contenu figuratif.. Il ne s'agit pas donc pas d'abord d'interpréter un quelconque contenu représentatif, comme dans la thérapie d'enfants non psychotiques, car leur production picturale se compose moins de traces figuratives dotées de significations latentes à décrypter, que de traces sensori-affectivo-motrices; loin de figurer des représentations préexistantes, ces traces vont au contraire conditionner la possibilité de l'accès à la représentation. Par ailleurs, l'intérêt thérapeutique de la médiation picturale dans la psychose consiste essentiellement en un travail de constitution des contenants psychiques, en lien avec la constitution d'un fond pour la représentation, avec une mise en place progressive des qualités plastiques de l'enveloppe psychique. 


\section{ARTIGO}

L'exemple de Tom, incapable de représenter un bonhomme au début de son atelier thérapeutique individuel de peinture, décrira les grandes étapes de la construction du fond pour cet enfant et introduira à la modélisation des dispositifs à médiation picturale dans la psychose et l'autisme:

Ce dispositif thérapeutique s'inscrit dans le cadre d'un hôpital de jour et comporte deux niveaux: celui de l'atelier thérapeutique individuel avec un soignant de l'institution, et celui de la supervision hebdomadaire, que j'assure en tant que psychologue institutionnelle. Je vais montrer comment le travail thérapeutique en médiation picturale s'articule autour de la constitution d'un fond pour la représentation: il s'agit d'une constitution des contenants psychiques, avec une mise en place des qualités plastiques de l'enveloppe psychique. Tom, âgé de sept ans au début de son atelier thérapeutique individuel de peinture, a l'allure d'un petit robot, sans affect, au désespoir des soignants qui ont l'impression de ne pas exister pour lui.

1. Au cours de ses premières séances de médiation picturale, Tom refuse de prendre le pinceau, qui «pique», pour peindre au doigt, «peau contre feuille», dans une sorte de cramponnement, avec la recherche d'un accolement peau contre peau. Tom exprime ainsi son lien à l'objet, lien d'agrippement forcené avec une terreur évidente du décramponnement. L'enfant devient une «sensation main agrippée à la feuille», sensation hallucinée très proche du pictogramme selon CastoriadisAulagnier (1975), comme un homme tombé dans un précipice et qui s'accroche à un rocher: il n'est que sensation paume de la main agrippée au rocher.

Il est alors incapable de représenter la moindre forme figurative, le fond pour la représentation n'est pas constitué, sa peinture se situe dans la position adhésive. Dans la position adhésive, il est impossible aux enfants de représenter une forme différenciée sur un fond, ils peuvent déchirer, perforer la feuille ou lancer des traces sans retour. En ce qui concerne l'enveloppe psychique, elle n'est pas constituée, l'enfant n'a pas intégré le sentiment d'enveloppe, et notamment la première enveloppe, de nature rythmique.

2. Les enfants sortent de la position adhésive par ce que j'ai proposé d'appeler la position de détachement du fond: un premier fond est constitué avec la possibilité cette fois d'un jeu entre figure et fond. Il s'agit non pas de formes figuratives ou représentatives mais formes sensori-affectivo-motrices. Cette étape correspond à une position symbiotique avec un fantasme de peau commune. Un décollement des 2 feuillets de la peau psychique apparaît, ainsi que la prise en compte de la tridimensionnalité. Chaque enfant aborde de façon singulière cette position, par des grattages, des collages et décollages, utilisation de la matière dans sa tridimensionnalité... Et pour Tom?

Quelque temps plus tard, il refuse d'utiliser la peinture pendant plusieurs mois. L'animatrice de l'atelier me dit incidemment que Tom dans le service ne 
cesse depuis quelque temps d'effectuer avec le bras une sorte de mouvement de balancier, bizarre. Je suggère d'exploiter ce mouvement corporel pour revenir à la peinture.

À l'atelier suivant, l'animatrice pose de grandes feuilles par terre, imite le mouvement de Tom, pinceau en main, sans toutefois peindre. Tom prend alors le pinceau qu'il enduit de peinture, et, va venir se coller à la référente, reprenant son mouvement de balancier du bras et cette fois de tout son corps, laisse de grandes traces en couleur de peinture sur la feuille à terre; l'animatrice accompagne leur mouvement commun des mots «balance, balançoire». Tom jubile.

Cette séance de peinture a inauguré pour Tom une période mutatrice de production picturale, il s'est mis à peindre régulièrement, seul sans l'appui de la thérapeute. C'est donc la relation transférentielle avec l'animatrice, dans le balancement rythmique partagé, qui permet à Tom de figurer des balayages rythmiques, un tracé qui met en jeu la séparation/retrouvailles avec la feuille. Dans le corps à corps rythmique avec la thérapeute, Tom ne fait plus des traces du registre de l'agrippement, ledécollage du support est possible pour y revenir, mais des traces-mouvements qui met en jeu la séparation/retrouvailles avec le support feuille.

Autrement dit, ce balancement automatique du bras a été interprété comme un message de non rencontre avec l'autre: ce n'est pas «je balance mon bras», ce n'est pas «je cherche à rencontrer d'autres bras», c'est «un bras se balance», il n'y a pas vraiment de message dans un bras se balance, c'est la thérapeute qui va le transformer en message en échoisant ce mouvement du bras: la référente a introduit un écho en double du mouvement qui devient partagé et créateur et, après coup, on pourrait dire que le message non encore advenu du bras qui se balance était de raconter l'histoire d'une rencontre non encore advenue avec la soignante, et que nous avons à l'interpréter comme la recherche d'une reprise du lien. Le message corporel ne devient paradoxalement un message que s'il est échoisé.

Tom a ainsi mis en place ce que Haag appelle le premier fond rythmique, ce qui a constitué une phase de la position de détachement du fond. Dans le dessin de l'enfant normal, Haag (1995) a observé que le bonhomme n'apparaît qu'après cette mise en place de traces rythmiques préfiguratives qui représentent les premiers échanges rythmiques avec la mère.

Tom a connu une seconde phase de détachement du fond: un an plus tard, à neuf ans,cet enfant change de soignant, un homme, qu'il tente à plusieurs reprises de frapper. Dans ce contexte, il initie une nouvelle façon de peindre, il prend son élan pour aller tamponner de toutes ses forces avec son pinceau une feuille suspendue au mur, il réalise des taches multicolores qui dégoulinent et il répète en criant au soignant: 


\section{ARTIGO}

«Je suis en colère! Ca éclate! Tais toi! Tu m'empêches!» ... Et chaque fois que ce soignant vient le chercher pour partir à son atelier thérapeutique de peinture, il lui répète: «Je t'aime ... Je vais taper sur la feuille». Du coup, il cesse de taper le soignant et ce processus rappelle l'élaboration première de la destructivité décrite par Winnicott: «Hé l'objet, je t'ai détruit. Tu comptes pour moi parce que tu survis à ma destructivité de toi. Puisque je t'aime, je te détruis tout le temps, dans mon fantasme inconscient» (Winnicott, 1971/1975, p. 125). En ce qui concerne Tom, il peut désormais différencier le registre du fantasme, autour du medium qu'il tamponne de toutes ses forces, de la réalité du lien avec le soignant, qu'il ne frappera plus.

3. C'est alors que Tom a pu accéder à la figuration humaine, après cette restauration d'un fond rythmique, dans le travail de la peinture et dans le transfert avec les soignants.

Dans le registre de la psychose, j'ai constaté que les premières apparitions de la figuration humaine, étaient souvent précédées du recours à une gestualité rythmique qui pouvait prendre la forme de frappes rythmées sur la feuille, de jeux d'écho gestuel avec l'animateur de l'atelier, de gestes d'éclaboussures en rythme ou de balayages rythmiques.

Pour la première fois depuis deux ans, Tom choisit une feuille noire comme support et il figure un "bonhomme de neige", avant de secouer son pinceau sur le bonhomme. Bonhomme d'abord sans visage, aux organes des sens raturés, comme chez Michaux, qu'il éclabousse ensuite à la peinture rouge en s'exclamant: "C'est de la colère! Il a de la colère sur les pieds!". Bonhomme de neige glacé comme Tom, mais qui s'anime avec une explosion de colère. Le fond rythmique des taches perdure, sous forme de giclures et d'éclaboussures sur les bonshommes.

À la séance suivante, il figurera encore un bonhomme blanc et rouge éclair surgi de la nuit en précisant: "Il est enfermé. Il ne peut pas avancer". Bonhomme qui semble en marche, avec grande force de présence, mais qui semble ne pas encore pouvoir faire visage.

Il lui faudra encore quelques mois de travail pour représenter l'intérieur du visage, puis des scènes, avec figuration de personnages et mise en histoire. Il a dès lors abordé la position de figuration ou de réflexivité (Roussillon, 2008), capacité de se voir, de se sentir et de s'entendre soi même, le deuxième fond se constitue, avec le dédoublement des lignes de terre et de ciel CF Haag (1995). Des formes représentatives à contenu figuratif ou imagé apparaissent. Cette étape correspond à la position dépressive selon M. Klein, elle se caractérise par l'emboîtement des deux feuillets de l'enveloppe psychique qui se constitue en Moi Peau et par l'entrée dans l'historicité et la quadrimensionnalité (1975). 
Le travail thérapeutique de Tom met en lumière le parcours des enfants psychotiques dans la médiation picturale, qui leur permet de construire un fond pour la représentation.

\section{La médiation conte: modalités sensorimotrices du transfert sur les personnages et sur l'histoire contée}

Avant de revenir à la médiation picturale, un détour par une autre médiation, la médiation conte, s'impose, du fait de la paradoxalité de cet exemple: le conte semble par excellence être une médiation verbale et il permet ainsi d'interroger particulièrement la question de la sensorimotricité. J'avancerai l'hypothèse qu'une appropriation du conte pour l'enfant psychotique et autiste ne deviendra possible qu'à l'appui du recours à ce registre sensorimoteur (Brun, 2012). D'ailleurs la plupart des ateliers thérapeutiques conte proposent aux enfants une scénarisation du conte par des jeux de rôle. La fonction du conte consistera avec des enfants psychotiques à mettre en place les formes primaires de symbolisation, formes sensorimotrices, qui pourront permettre dans un temps ultérieur seulement d'accéder aux contenus symboliques du conte, médiés par le langage verbal. De façon générale, la symbolisation à partir d'un atelier thérapeutique conte s'effectuera pour l'enfant dans une problématique psychotique d'abord à partir des éléments perceptifs et sensoriels du cadre-dispositif, comme les objets du décor, les costumes, les marqueurs spatiotemporels, les rythmes répétitifs, ainsi que des réponses sensorimotrices, et non pas exclusivement verbales, des soignants.

Les modalités transférentielles dans un groupe conte s'interprètent habituellement à partir des identifications des enfants aux personnages du conte. Ce processus identificatoire paraît souvent problématique chez les enfants psychotiques: l'identification aux personnages s'effectue souvent de façon adhésive, l'enfant n'assimile pas tel ou tel trait du personnage, il devient le personnage auquel il s'identifie corporellement, dans une relation spéculaire. Ainsi tel enfant tombe de sa chaise quand le loup tombe dans la cheminée, il ne visualise pas le loup, il devient le loup. Ce type de relation de spécularité (Castoriadis-Aulagnier, 1975) s'effectue ici encore à partir de la réactivation de sensations hallucinées, par exemple ici de chute. Le travail thérapeutique à partir du conte suscite en effet un retour hallucinatoire de sensations ou d'angoisses catastrophiques et ces sensations hallucinées vont prendre forme dans la scénarisation pour devenir ainsi figurables et transformables. 


\section{ARTIGO}

L'enfant peut accompagner par exemple le souffle du loup pour détruire la maison des petits cochons de coups de pied sur le mur, il éprouve ainsi corporellement la résistance du mur, qui lui permet de ne pas céder à l'angoisse d'effondrement. L'enfant tente ainsi de représenter des terreurs primitives à partir de sa gestualité et souvent de bruitages et il donne, à l'appui d'images du conte, une forme imagée à ces terreurs irreprésentables.

Ces formes sensorielles et motrices de la symbolisation, qui passent par le corporel, relèvent souvent de la dynamique de l'image motrice, conceptualisée par Freud, qui la définit comme une image sensorielle avec perception de mouvement, ou du signifiant formel décrit par Anzieu (1987) comme impression corporelle constituée d'images tactiles, kinesthésiques, posturales ou proprioceptives.

\section{Les thérapeutes: mimogestualité et théâtralisation}

Les thérapeutes utilisent aussi beaucoup le langage du corps, la mimogestualité et la théâtralisation. Le conteur doit interpréter son récit par ses mimiques expressives, par sa gestualité et par ses postures, ce qui constitue un support important pour des enfants en difficulté avec le langage verbal: ces derniers ont besoin en quelque sorte d'une traduction en langage corporel, qui sera aussi un enclencheur de jeu théâtral pour les enfants. Cette dynamique mimogestuoposturale restitue la chorégraphie première entre la mère et l'enfant et propose une interprétation à l'enfant du conte qui passe par un autre sujet: la mimogestualité du conteur a ainsi une fonction de révélateur des affects. Il dispose de sa voix, de son regard et de ses mimiques en rapport avec le contenu du conte pour solliciter ou rassembler l'attention des enfants. Par ailleurs, le conteur joue sur la modulation de son intonation, qui participe à la (re)constitution d'une sorte de matrice phonique primordiale, de "chair phonique" selon une expression d'Artaud, dans laquelle l'intonation de la voix porte la signification et offre une vocalité du sens, préalable ou coextensive à l'intelligibilité des contenus verbaux.

Les thérapeutes à la disposition des enfants pour le jeu de rôles postérieur au contage auront souvent une fonction de doublage: ils se mettront par exemple derrière ou à côté des enfants, en appui sur leur mimogestualité qu'ils accompagneront dans le meilleur des cas, ou initieront pour être eux-mêmes ensuite doublés par les enfants: ils doubleront l'enfant qui les doublera aussi, restaurant dans ce processus la fonction première de miroir en double (Roussillon) de l'objet primaire, souvent défaillante dans le registre de la psychose.

Avec des enfants autistes, on gagnera à accentuer la matérialité des éléments du cadre pour pouvoir jouer sur une possible intégration sensorielle, défaillante 
dans la pathologie autistique, caractérisée par la dissociation des différentes composantes de la sensorialité. La conception du dispositif suivant vise à solliciter la symbolisation sensorimotrice dans tous les registres sensoriels et à déployer des transpositions sensorielles d'une modalité à l'autre, du visuel, au tactile, à l'auditif.

Par exemple le conte est traditionnellement rythmé par des formulettes et bouts-rimés, que les soignants peuvent éventuellement accompagner de gestes répétitifs: ils effectuent ainsi des transpositions sensorielles du registre sonore au registre gestuel, ce qui est particulièrement important pour les enfants autistes qui souffrent du démantèlement (Meltzer, 1975), autrement dit d'une dispersion de l'attention qui sépare les capacités perceptuelles, sans accès à la consensualité. Comme l'a montré D. Stern (1985/1989), la transposition d'un mode d'expression dans une autre modalité sensorielle, par exemple entre les registres kinesthésique, sonore, visuel, mimogestuopostural, caractérise les phénomènes d'accordages entre la mère et l'enfant.

Dans cette perspective, la restauration de ces jeux théâtralisés de la première enfance semble une des spécificités des jeux de rôle, qui rappellent certaines modalités du psychodrame: l'objectif majeur consiste à transformer la destructivité des enfants grâce aux jeux théâtralisés et le conte sera un enclencheur du jeu.

On pourra par exemple intervenir quand un enfant donne un coup de pied, en demandant en quel personnage du conte il s'est transformé, invitant ainsi l'enfant au faire semblant et dédramatisant sa violence. Si un enfant dit par exemple «je vais t'exploser, te tuer», le thérapeute peut resituer ces paroles dans le cadre du conte, en disant: «c'est le petit cochon qui dit cela au loup», identifiant l'enfant au courageux petit cochon plutôt qu'au méchant loup. Les thérapeutes peuvent aussi utiliser leur mimogestualité pour doubler de façon théâtrale les gestes destructifs de l'enfant, en évoquant telle ou telle séquence du conte: c'est encore une façon de restaurer la relation de miroir en double de l'enfant avec son environnement.

Haag (2000) rappelle que dans le développement normal de l'enfant, autour de six mois, la théâtralisation joyeuse de la mère, le faire-semblant permet la transformation et l'intégration de la violence pulsionnelle érotique du bébé; elle transforme ainsi la griffe en caresse, la morsure en baiser et permet la mise en fantasme de la partie destructrice, par exemple dans le fameux jeu du lion, qui théâtralise dans le plaisir réciproque le fantasme d'incorporation.

Le processus thérapeutique dans les groupes conte évolue généralement d'une position adhésive à une position de figuration scénique, au moment où les enfants commencent à mettre en forme leurs angoisses, puis à une position de scénarisation, 


\section{ARTIGO}

associée à la capacité réflexive, qui se définit comme la projection et la mise en scène de scénarios fantasmatiques dans l'espace du conte.

Pour conclure, le travail thérapeutique au sein de cadres-dispositifs à médiation amorce un accès à la figurabilité par un processus de passage du registre sensori-moteur au figurable. Un des enjeux principaux des médiations thérapeutiques dans les cliniques de la psychose consiste à pouvoir ainsi faire advenir à la figuration des expériences primitives non symbolisées, d'ordre sensori-affectivo-moteur. Le travail de mise en forme du medium va en effet susciter des messages corporels, visuels, kinesthésiques, mimo-gestuo-posturaux, qui vont pouvoir prendre sens dans les interrelations au sein de l'atelier, avec les thérapeutes mais aussi avec le groupe d'enfants. Il s'agira donc de rendre signifiants, dans la restauration de la relation primaire à l'objet, les mouvements, les sensations, les vécus corporels d'ordre cénesthésique, kinesthésique, mimogestuo-postural, de donner une valeur de message aux éprouvés de l'enfant psychotique, notamment par le partage d'affects, et de les doter d'un sens partageable avec les thérapeutes et le groupe, processus au cours duquel les sensations pourront progressivement se transformer en émotions. À travers la sensorialité, la motricité, le mouvement, le processus thérapeutique consiste donc à mettre en forme, en figure, en rythme, des impressions sensorielles, qui vont pouvoir devenir des représentations-choses sensorielles, que l'enfant pourra s'approprier dans un processus de réflexivité.

Comment les dispositifs à médiation peuvent-ils enrichir la théorie psychanalytique et notre pratique dans le cadre traditionnel de l'analyse d'adultes? La recherche exposée précédemment montre combien les médiations thérapeutiques dans le champ des cliniques de l'extrême peuvent enrichir notre pratique dite traditionnelle, en nous engageant à prendre en compte, dans la cure ou dans la psychothérapie psychanalytique, les formes du langage sensorimoteur et la problématique de l'intersubjectivité. Il s'agit aussi d'explorer les formes fondamentales de la psychopathologie à l'appui de la modélisation des différents dispositifs de soin à médiation accueillant les cliniques des souffrances identitaires et les pathologies graves du narcissisme.

\section{References}

Anzieu, D. (1987). Les signifiants formels et le Moi Peau. In D. Anzieu, D. et al. Les enveloppes psychiques (pp. 1-22). Paris: Dunod.

Brun, A. (2007). Médiations thérapeutiques et psychose infantile. Paris: Dunod (réédit revue et augmentée, 2010). 
Brun, A. (2009). Mediaciones terapeuticas y psicosis infantil. Barcelone: Herder.

Brun, A. (2012). La médiation thérapeutique du conte dans la psychose infantile et dans l'autisme. In R. Kaës (Dir.), Contes et divans, pp. 193-214.

Brun, A., Chouvier, B., Roussillon, R. (2013). Manuel des médiations thérapeutiques. Paris: Dunod.

Castoriadis-Aulagnier, P. (1975). La violence de l'interprétation. Paris, PUF.

Chouvier, B. et coll. (2002). Matière à symbolisation. Lausanne: Delachaux et Niestlé.

Haag, G. (1995). La constitution du fond dans l'expression plastique en psychanalyse de l'enfant. Sa signification dans la constitution de la psyché. In S. Decobert, F. Sacco et coll., Le Dessin dans le travail psychanalytique avec l'enfant (pp; 63-87). Ramonville Saint-Agne: Érès.

HaAG, G. (2000). Propositions pour la compréhension des différentes formes de violence chez le jeune enfant. In B. Lacroix, M. Monmayrant, Enfants terribles, enfants féroces (pp. 177-190). Ramonville Saint-Agne: Érès.

Kaës, R. (2000), L'Appareil psychique groupal: constructions du groupe. Paris: Dunod. (Travail originel publié dans 1976).

Meltzer, D. et coll. (1975). Explorations dans le monde de l'autisme. Paris: Payot.

Milner, M. (1979). Le rôle de l'illusion dans la formation du symbole. Revue française de psychanalyse, 5-6. Repris in B. Chouvier (Eds.), Matière à symbolisation, art, création et psychanalyse. Paris: Delachaux et Niestlé, 1998. (Travail originel publié dans 1955).

Roussillon, R. (2001). Le plaisir et la répétition. Paris: Dunod.

Roussillon, R. (2008). Le transitionnel, le sexuel et la réflexivité. Paris: Dunod.

Stern, D. (1989). Le monde interpersonnel du nourrisson. Paris: PUF. (Travail originel publié dans 1985).

Winnicott, D. W. (1975). Jeu et réalité. L'espace potentiel. Paris: Gallimard. (Travail originel publié dans 1971).

Winnicott, D. W. (2000). La crainte de l'effondrement et autres situations cliniques. Paris: Gallimard. (Travail originel publié dans 1974).

\section{Resumés}

(O encontro analítico nos dispositivos das mediações terapêuticas, nos limites de uma análise)

As mediações terapêuticas em práticas institucionais dependem da psicoterapia psicanalitica quando se baseiam na análise da transferência e da associatividade psíquica no âmbito do método analítico. Apoiando-se na experiência clínica da psicose infantil, este artigo questionará as modalidades específicas do desenvolvimento 


\section{ARTIGO}

dos processos de simbolização, considerando nomeadamente a associatividade sensório-motora.

Palavras-chave: Associatividade sensorio-motora, metapsicologia das mediações terapêuticas. sensório-motricidade, simbolização primária

(The analytic encounter in therapeutic mediation devices, at the limits of an analysis)

Therapeutic mediations in institutional practices fall within psychoanalytic psychotherapy when they take into account transference and psychic associativity at the very foundation of the analytic method. Based on the clinical experience of infantile psychosis, this article questions the specific terms of symbolization processes that unfold, in particular from the consideration of sensorimotor associativity.

Keywords: Sensorimotor associativity, metapsychology of therapeutic mediations, sensori-motivity, primary symbolization

(El encuentro analítico en los dispositivos de las mediaciones terapéuticas, en los confines de un analisis)

Las mediaciones terapéuticas en prácticas institucionales dependen de la psicoterapia psicoanalítica cuando se basan en el análisis de la transferencia y de la asociatividad psíquica en el ámbito del método analítico. Apoyándose en la experiencia clínica de la psicosis infantil, este artículo cuestionará las modalidades específicas del desarrollo de los procesos de simbolización, considerando, sobre todo, a la asociatividad sensoriomotora.

Palabras claves: Asociatividad sensoriomotora, metapsicología de las mediaciones terapéuticas, sensoriomotricidad, simbolización primaria

(Die analytische Begegnung in Systemen zur therapeutischen Mediation, an den Grenzen einer Analyse)

Therapeutische Mediationen in der institutionellen Praxis hängen von der psychoanalytischen Psychotherapie ab, wenn sie auf der Transfer-Analyse und der psychischen Assoziation begründet sind, im Bereich der analytischen Methode. Basierend auf der Behandlung kindlicher Psychosen hinterfragt dieser Artikel die besonderen Modalitäten der dort eingesetzten Symbolisierungsverfahren, insbesondere ausgehend von der Berücksichtigung der sensomotorischen Assoziation.

Schlüsselwörter: Sensomotorische Assoziation, Metapsychologie der therapeutischen Mediationen, sensomotorische Koordination, primäre Symbolisierung 
（用精神分析法对儿童精神病进行干预性治疗和精神分析法的局限性）

通常人们对儿童精神病进行干预性治疗时, 主要依靠的是精神分析与心理治 疗法, 他们都基于精神分析法中的心理移情法和感统关联法。依托童年精神病的 临床经验, 这篇文章质疑精神分析的象征化进程 (processos de simbolização, 特别针对感统联系疗法中出现的一些问题进行探讨。

关键词: 感觉-驱动关联, 心理学干预治疗, 感统训练(sensorio-motricidade), 初 始象征化 (simbolização primária)。

Citação/Citation: Brun. A. (2015, junho). La rencontre analytique dans les dispositifs à médiations thérapeutiques, aux limites del l'analyse. Revista Latinoamericana de Psicopatologia Fundamental, 18(2), 234-252.

Editores do artigo/Editors: Prof. Dr. Manoel Tosta Berlinck e Profa. Dra. Sonia Leite

Recebido/Received: 23.04.2014/ 04.23.2014 Aceito/Accepted: 15.6.2014 / 6.15.2014

Copyright: (C) 2009 Associação Universitária de Pesquisa em Psicopatologia Fundamental/ University Association for Research in Fundamental Psychopathology. Este é um artigo de livre acesso, que permite uso irrestrito, distribuição e reprodução em qualquer meio, desde que o autor e a fonte sejam citados / This is an open-access article, which permits unrestricted use, distribution, and reproduction in any medium, provided the original authors and sources are credited.

Financiamento/Funding: A autora declara não ter sido financiada ou apoiada / The author has no support or funding to report.

Conflito de interesses/Conflict of interest: A autora declara que não há conflito de interesses / The author has no conflict of interest to declare.

\section{Anne Brun}

Professeur de Psychopathologie et Psychologie Clinique. Directrice du CRPPC (Centre de Recherche en Psychopathologie et Psychologie Clinique), Université Lumière Lyon 2. université Lyon 2, CRPPC,

5 avenue Pierre Mendès

France, 59676, Bron Cedex.

e-mail: annebrunlyon@orange.fr 